\title{
Digital Chest Radiography With Storage Phosphor Systems: Potential Masking of Bilateral Pleural Effusions
}

\author{
Harrell G. Chotas and Carl E. Ravin
}

\begin{abstract}
A photostimulable storage phosphor (PSP) computed radiography imaging system was analyzed for its potential to mask pleural effusion during normal image processing. This phenomenon has been observed in several clinical cases in our hospital. To better understand the relationship between pleural effusion and the PSP radiograph appearance, portable radiographs of an anthropomorphic chest phantom were acquired with the PSP system in conditions simulating various quantities and distributions of pleural fluid. It was observed that the optical density of the film in one hemithorax was significantly influenced by whether or not fluid was present in the opposite hemithorax. This optical density dependence was determined to be a system-induced effect that results from the image processing (histogram analysis) technique used by the PSP system during image plate readout. It is important to recognize that the PSP system's normal optical density (sensitivity) adjustment can obscure the presence of bilateral pleural fluid accumulation, particularly if the opposite hemithorax contains fluid in an equal or greater amount.
\end{abstract}

Copyright $₫ 1992$ by W.B. Saunders Company

KEY WORDS: photostimulable phosphor, storage phosphor, computed radiography, pleural effusion.

$\mathbf{P}$ HOTOSTIMULABLE storage phosphor (PSP) computed radiography imaging systems were first commercially introduced in 1983, and since that time over 400 systems have been installed worldwide. The benefits to be derived from the use of PSP imaging systems in portable chest radiography have been well documented. ${ }^{1,2}$ Among the advantages of the system is the ability to produce radiographs that have excellent lung-image contrast despite wide variation in x-ray exposure technique. The capability of the PSP system to dynamically adapt itself in response to differences in patient size and $\mathrm{x}$-ray technique virtually eliminates the need for repeat examinations due to under- or over-

From the Department of Radiology, Thoracic Imaging Research Division, Duke University Medical Center, Durham, NC.

Address reprint requests to Harrell $G$. Chotas, MS, Depart ment of Radiology, Box 3302, Duke University Medical Center, Durham, NC 27710.

Copyright 1992 by W.B. Saunders Company

0897-1889/92/0501-0005\$03.0010 exposure, a feature that is particularly important in bedside (portable) chest $\mathrm{x}$-ray examinations.

One important requirement that must not be overlooked in the design and development of digital imaging technologies is that the techniques used by the system to achieve this "contrast optimization" should not alter the image in such a way as to diminish or enhance the appearance of large area opacities that may occur in one or both hemithoraces. Recently we have noted several clinical cases of pleural effusion in which there was asymmetric pleural fluid accumulation in the two hemithoraces. Two such cases are illustrated in Figs 1 and 2. In each case, the fluid in the less affected hemithorax was not detectable in the PSP radiographs that were acquired in the supine view, although the fluid was confirmed in the decubitus views and/or with computed tomography (CT). Those observations led to this investigation: to observe the effects of pleural fluid accumulation on optical densities in portable PSP chest radiographs, and to determine the extent to which the radiographic appearance of one hemithorax is influenced by the radioopacity of the other hemithorax.

\section{MATERIALS AND METHODS}

\section{Storage Phosphor System}

The PSP imaging system used in this investigation is a Philips Computed Radiography system (PCR 901, Philips Medical Systems North America, Shelton, CT) which has been described in detail elsewhere. ${ }^{3}$ Briefly, the PSP system is based on a reusable storage phosphor imaging plate that serves as an area detector which, in its cassette, may be used with conventional $x$-ray equipment in place of the traditional film-screen detector. The imaging plate has an extremely wide sensitivity range (latitude) that spans four orders of magnitude, and a linear response over the entire range. When exposed to $x$-rays, part of the incident radiation is stored as a latent image on the imaging plate. This image information is then digitized by an automated plate readout system which uses a scanning laser beam to stimulate the energy trapped in the plate and a photodetector to measure the light energy emitted by the plate at each pixel location. The resulting digital image matrix $(1760 \times 2140 \times 10$ bits) is then further processed (grayscale- and/or edgeenhanced) by the PSP system for display on laser-printed film or at video workstations.

Because the imaging plate is inherently a very wide 

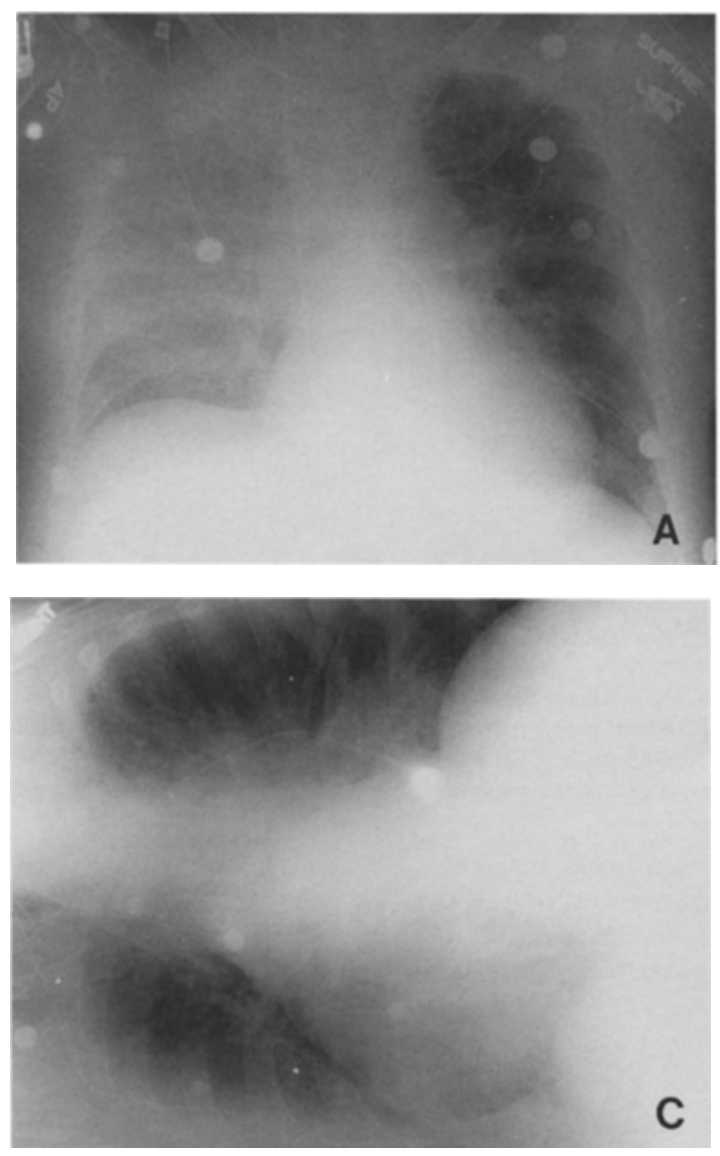

latitude detector, the readout operation actually occurs in two stages in normal clinical operations. First, a lowintensity, low-resolution $(256 \times 256$ pixels $)$ "preread" operation is performed to determine the operating parameters (laser power and photomultiplier gain) that are then used during the second, main read operation. The preread step, also called "detector autoranging," is based on histogram analysis of the preread image and is intended to guarantee that the clinically useful data are appropriately mapped to the available display (pixel) range. The effectiveness and accuracy of the readout system in performing the preread operation is critical to this investigation, for it is at this stage that the image data are scaled to the range that is used in forming the final image display.

\section{Image Acquisition}

Portable anterjor-posterior (AP) chest radiographs of an anthropomorphic chest phantom (Humanoid Systems, Carson, CA) were acquired in four phantom configurations, described below. In each phantom arrangement, two PSP images were acquired separately using $36 \times 43-\mathrm{cm}(14 \times 17$ inch), ST-III imaging plates. In each exposure pair, one of the imaging plates was processed normally using the PSP system's "Portable Chest X-ray" operating mode. In this "clinical mode" of operation, the PSP plate readout system performs the detector autoranging step described above

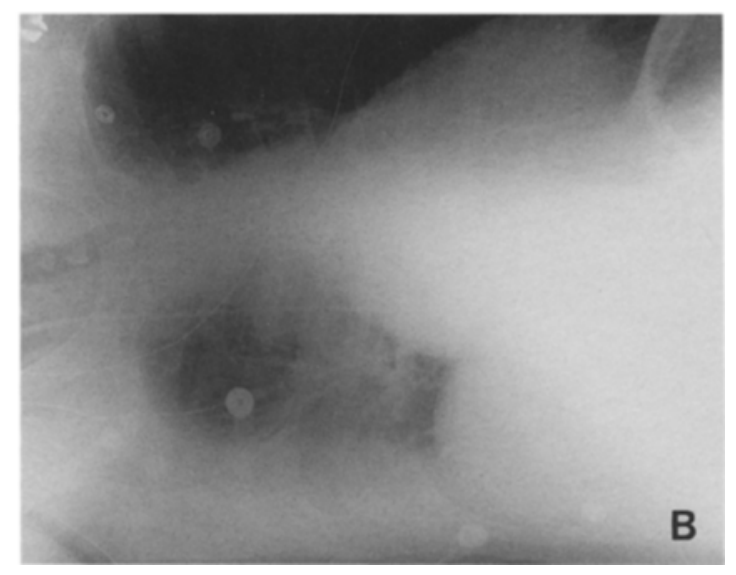

Fig 1. Bilateral asymmetric pleural effusion confirmed with decubitus views. (A) Anteroposterior supine chest radiograph demonstrates generalized increased opacity over the right hemithorax suggesting the diagnosis of a large, freely flowing, right pleural effusion. Opacity of the left hemithorax was judged to be normal and there was no suspicion of a left pleural effusion. (B) Right side down lateral decubitus projection confirms the suspected freely flowing right pleural effusion. (C) Left side down lateral decubitus position confirms a smaller, freely flowing, left pleural effusion.

prior to the main read operation, and the final image data are thereafter restricted to the exposure range that is selected by that subsystem. The second PSP image in each pair was processed in a fixed-latitude, fixed-sensitivity mode of the PSP system (detector latitude $L=4.0$; sensitivity $S=200$ ), a mode that bypasses the preread step and uses the full sensitivity range of the detector. In all cases, the elapsed time between plate exposure and readout was approximately 60 minutes.

All radiographs were made using a portable $\mathrm{x}$-ray system (GE AMX-4, General Electric Medical Systems, Waukesha, WI) at $100 \mathrm{kV}$ with $3 \mathrm{~mm}$ Aluminum filtration, $1.0 \mathrm{mAs}$, 48 inch focus-film distance, and no antiscatter grid. These imaging conditions are typical of those used in our hospital for portable chest $x$-ray examinations.

Four phantom configurations were used to simulate different clinical conditions: normal, mild unilateral-, mild bilateral-, and asymmetric-pleural fluid accumulations. The normal condition was simulated using the chest phantom with an acrylic sheet $(36 \times 43-\mathrm{cm}, 1 \mathrm{~cm}$ thick) attached to the anterior face of the phantom, thereby providing a horizontal surface on which fluid containers could rest. In the remaining phantoms, pleural fluid was simulated by placing a polyethylene bag containing saline $(0.9 \% \mathrm{NaCl})$ on the phantom over a lung region. In the projected radiograph, each saline bag completely covered a single 

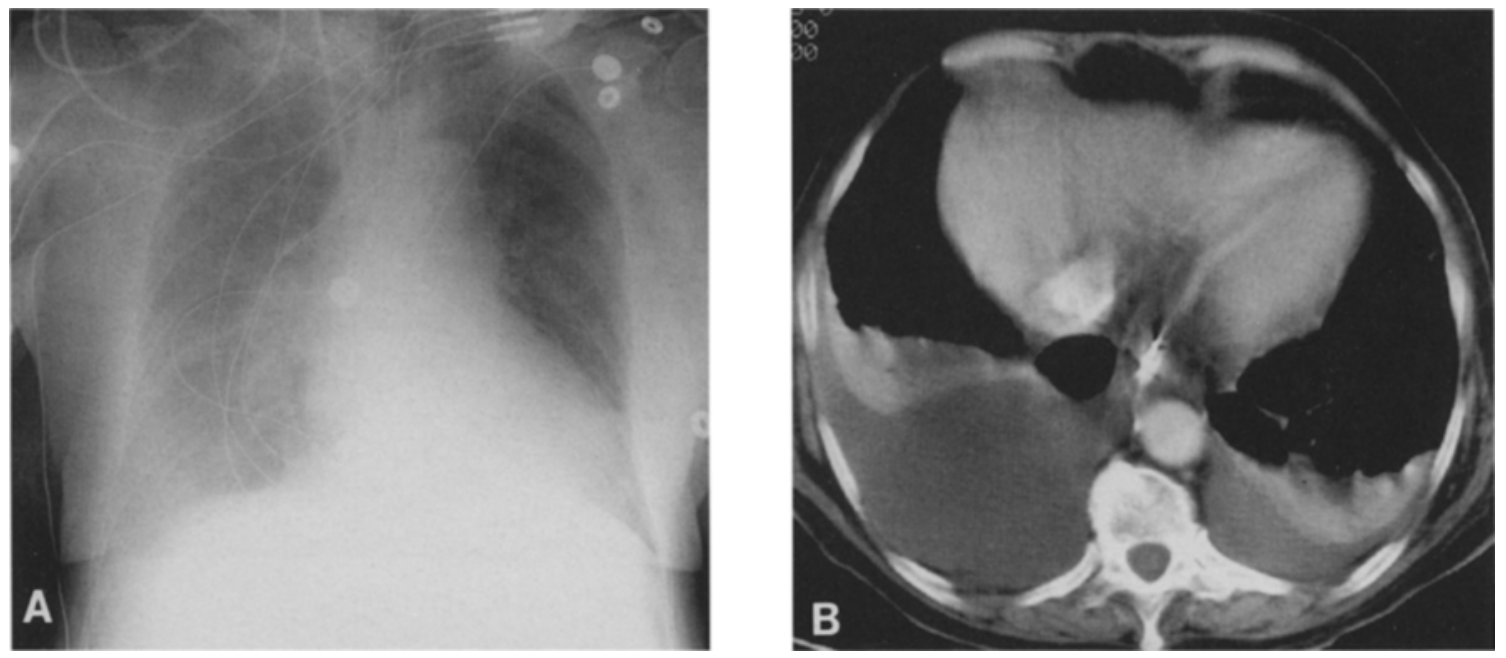

Fig 2. Bilateral asymmetric pleural effusion with CT confirmation. (A) Anterposterior supine chest radiograph demonstrating generalized increased opacity over the right hemithorax compatible with a posteriorly laying right pleural effusion. The opacity of the left hemithorax was judged normal and no pleural effusion was suspected on the left. (B) Selected cut from a CT examination on the same patient confirms the suspected large right pleural effusion but also demonstrates a smaller, freely flowing, left pleural effusion.

lung, with only minimal overlap of the adjacent subdiaphragm or chest wall regions. Table 1 shows the fluid quantities used over each lung in each phantom configuration. It should be noted that in all but the normal phantom configuration, the left hemithorax "contained" a constant $500 \mathrm{ml}$ of fluid; only the fluid over the right hemithorax was varied.

\section{Image Analysis}

Optical densities were measured in the four laser-printed films that were acquired in the clinical mode of the PSP system. Each clinical chest film generated by the PSP system in our hospital contains two versions of the chest image, one resembling a well-exposed conventional radiograph and the other having significant contrast and edge enhancement to provide information not available in the first image. In this investigation, optical density was measured in the "pseudoconventional" film image as it is normally processed and printed in our hospital for clinical use, simulating a medium latitude conventional film, as previously reported ${ }^{4}$ (PCR system parameters: rotation amount $=0.9$, contrast type $=\mathrm{D}$, rotation center $=1.5$, density shift $=0.15$, with

Table 1. Phantom Descriptions

\begin{tabular}{lcc}
\hline & \multicolumn{2}{c}{ Quantity of Fluid (ml) } \\
\cline { 2 - 3 } Phantom Configuration & Right Hemithorax & Left Hemithorax \\
\hline Normal & 0 & 0 \\
Mild unilateral pleural effusion & 0 & 500 \\
Mild bilateral pleural effusion & 500 & 500 \\
Asymmetric pleural effusion & 1,500 & 500 \\
\hline
\end{tabular}

NOTE. Polyethylene bags containing saline (quantities shown above) were used to simulate pleural fluid in the anthropomorphic chest phantom. Note that left hemithorax fluid volume remained constant in all pleural effusion configurations $\$ 500$ $\mathrm{mll}$. slight spatial frequency enhancement at $0.25 \mathrm{C} / \mathrm{mm}$ ). The more enhanced image was not used.

Six optical density measurements were made in each film image using a TA-TBX densitometer (Tobias Associates,

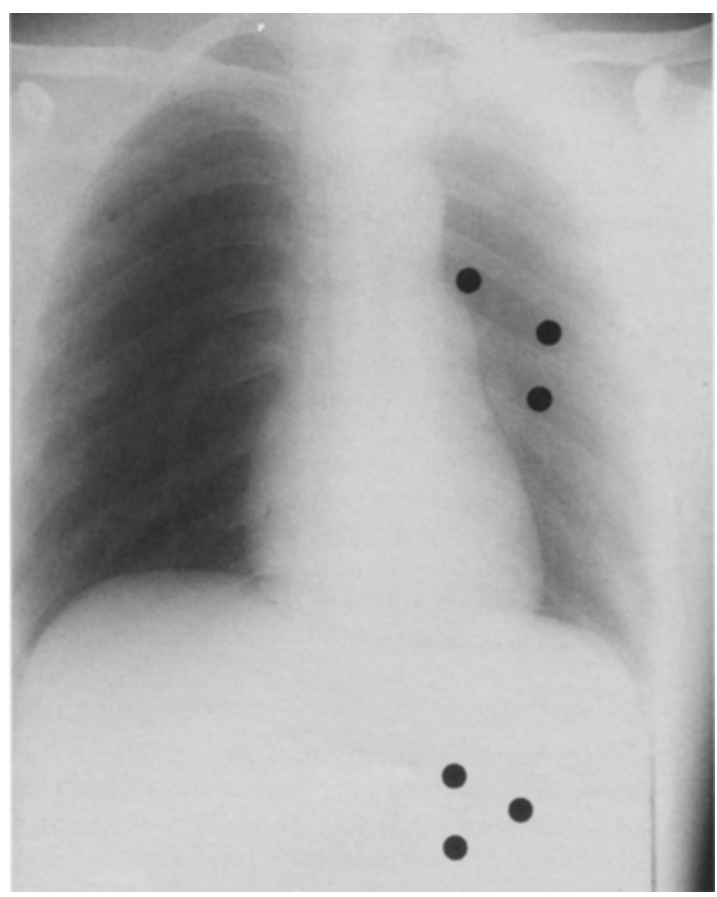

Fig 3. Optical density measurement sites. Shows the image locations at which optical densities were measured in laser-printed film radiographs of a chest phantom acquired in the PSP system's clinical mode. The "unilateral pleural effusion" phantom configuration is shown. 
Ivyland, PA). The same measurement sites were used in each phantom image, with three samples each in the left lung and subdiaphragm regions (Fig 3 ). The average density in each region was calculated and plotted. Sample points were selected on the basis of small-area uniformity (to minimize measurement uncertainty) and were not intended to represent a meaningful "average" density of the anatomical region. Measurement sites in the lung were selected from among the darker inter-rib spaces.

To supplement film analysis and to gain insight into the PSP readout system performance, unprocessed digital image data (without grayscale modification or edge enhancement) from all eight PSP images were transmitted to our image processing network for analysis. The histogram of each image was computed and plotted to illustrate the distribution of pixel values in each image. In the nonclinical, fixed mode images, pixel values are directly related to the $x$-ray exposure intensity detected at each location on the imaging plate. Histograms of these fixed mode images, therefore, illustrate the full range of exposure data that was available to the readout system when processing the four corresponding "clinical mode" images. In the clinical mode images, only a fraction of the system's sensitivity range is used during the image readout process, and resultant image pixel values determine the assignment of grayscale values (optical densities) in the final image display. A comparison of the histograms of the clinical mode and fixed mode images shows the exposure data subrange that was selected and mapped to pixel values by the readout system in each phantom image and illustrates the effect that this can have on image optical density.

\section{RESULTS}

Average optical density measurements in each region of the four clinical mode PSP radiographs are shown in Fig 4. It can be seen that, although there was no physical change in left hemithorax opacity (quantity of fluid was constant), the optical density in the left hemithorax image region increased as the quantity of fluid over the other (right) hemithorax increased. Similarly, there was a corresponding increase in subdiaphragm optical density in the bilateral and asymmetric pleural fluid conditions, relative to the other two cases in which the right hemithorax was unobstructed.

Photostimulable storage phosphor image histograms are presented in Fig 5. Selected anatomical pixel ranges are noted for the normal phantom, and the range of pixel values observed in the left lung region of each image is indicated in each plot. In the PSP system, lower pixel values correspond to higher $\mathrm{X}$-ray intensity and higher film optical density (in a standard negative image), as shown. In all four clinical mode images, the same effective detector latitude was automatically selected by the PSP readout system for acquisition during the main read operation $(\mathrm{L}=1.6)$. This is the minimum detector latitude permitted by the digital imaging system when digitizing chest images.

Changes in the distribution of image pixels resulting from alterations in phantom pleural fluid can be noted in both the clinical and fixed mode histograms (Fig 5). Generally, as more fluid was added over the lung regions, the range

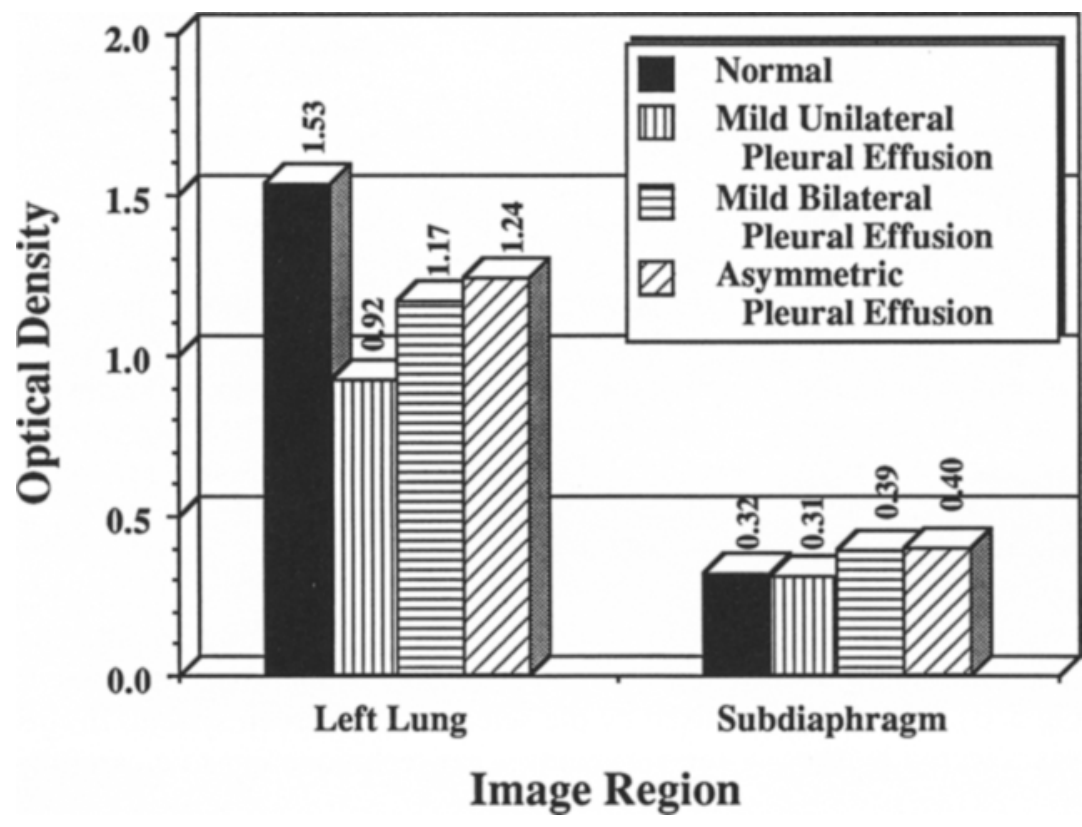

Fig 4. Film optical density. Average optical density values plotted by chest region for each phantom configuration $( \pm 5 \%)$. Although the radioopacity (fluid quantity) in the left hemithorax was held constant except in the normal phantom condition, optical density in that region increased as the opacity of the right hemithorax increased. Optical density in the subdiaphragm region also increased when fluid was present over both hemithoraces. 

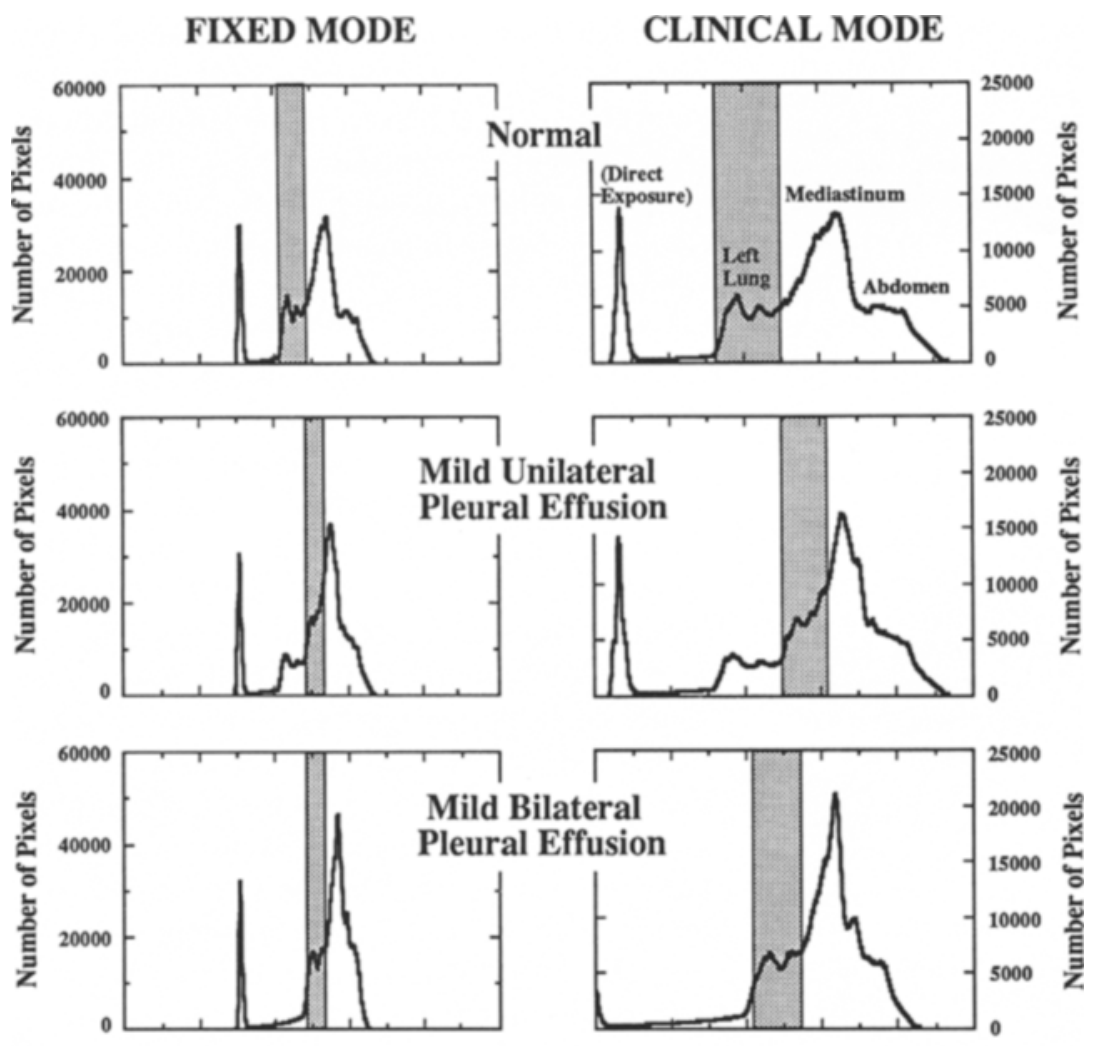

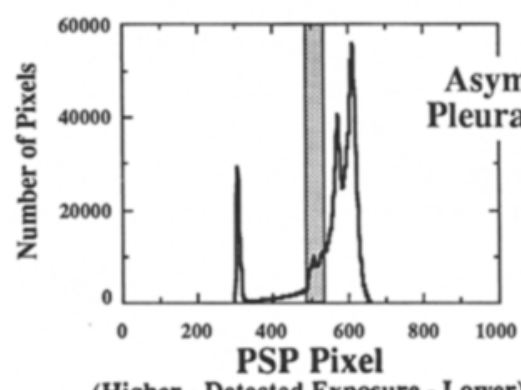

(Higher - Detected Exposure - Lower)

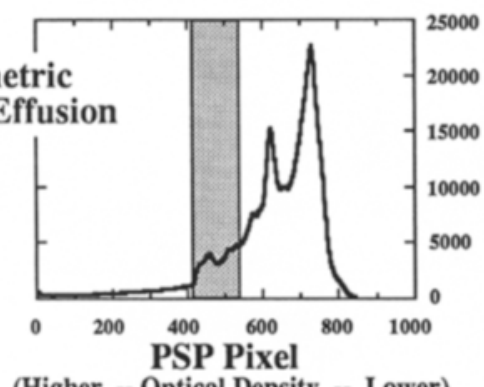

(Higher -. Optical Density -- Lower)

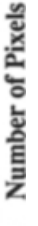

Fig 5. Photostimulable phosphor image histograms. Illustrates the distribution of pixel values in each PSP image. Shaded areas illustrate the approximate pixel ranges found in the left lung region. (A) Fixed mode images. No significant change in left lung pixel values in the three pleural effusion (PE) phantoms; (B) Clinical mode images. Pixel range in left lung varied with $P E$ phantom configuration, and approached the "normal" range when fluid over right lung region was present. of $x$-ray intensities transmitted through the phantom became narrower and the histogram peaks became sharper, as would be expected. The fixed mode histograms (Fig 5 A) demonstrate that the exposure range detected in the left lung region (shaded section) did not change significantly as fluid quantity over the right hemithorax increased. Histograms of the clinical mode images, however, show that the particular exposure ranges selected by the PSP system for final image processing were significantly affected by the introduction of additional fluid over the right hemithorax (Fig $5 \mathrm{~B}$ ). Although the same detector latitude was selected by the system in all four clinical mode images, the exact locations of the selected exposure windows were affected by the changes in the distributions of $x$-ray intensities incident on the imaging plates. This resulted in a downward shift in left hemithorax pixel values in the clinical mode images, which, in turn, led to the observed film optical density shifts.

\section{DISCUSSION}

In traditional film-screen radiography, the latitude and sensitivity of the image detector is fixed by the selected film-screen system. In this environment, $x$-ray technique must be carefully 
controlled (by the technologist or a phototimer) to ensure that the film is optimally exposed to properly use the available contrast range of the film. With the advent of storage phosphor imaging systems, however, radiologists have gained the ability to effectively tune the image detector to the patient so that image contrast can be optimized in every image. Whereas this capability has been shown to be extremely effective in reducing the number of unacceptable films, detector systems with variable latitude and sensitivity (such as the PSP system described in this investigation) may introduce film density changes in one image region due to physical changes in a different chest region, and this can be misleading to the radiologist and make diagnosis more difficult. This problem is particularly relevant in clinical conditions involving large-area pleural opacities (eg, fluid), because in these cases the distribution and possibly the dynamic range (latitude) of the radiation transmitted by the patient is altered, which can trigger a significant optical density shift. Similarly, such factors as patient position and field of view (both of which affect image composition) can also affect image latitude.

The potential masking of asymmetric bilateral pleural effusions is not unique to digital imaging and is certainly possible with conventional imaging techniques, particularly if such films are incorrectly exposed. However, because $\mathrm{x}$-ray techniques used in conventional imaging are selected on the basis of body habitus without preexisting knowledge of underlying pathology, an appropriately exposed conventional film would be expected to show increased opacity over both hemithoraces reflecting the bilateral nature of the pleural effusions. In computed radiography, the final lung optical density is automatically reset in such a fashion that the hemithorax with the smaller pleural effusion is made to appear "normal," and consequently the potential to fail to recognize that an abnormality may exist on the "normal appearing" side is increased.

The degree to which optical densities in computed radiography images are affected by plate reader-induced changes in image pixel values is directly related to the image postprocessing (display) parameters selected and used at each institution. In our hospital, PSP chest radiographs are processed for display such that image contrast resembles that of a mediumlatitude conventional film. ${ }^{4}$ Changes in the system's image display parameters can increase or decrease the likelihood of pleural effusion masking.

From a clinical standpoint, it is important to recognize that the image processing techniques used by computed radiography units can obscure the presence of pleural effusion on one side, particularly when there is a significantly larger effusion on the opposite side. Confusion is created in that one may inadvertently consider only diagnoses appropriate to unilateral pleural effusions rather than recognizing the bilateral nature of the process. In such clinical situations, decubitus films and/or CT analysis may be useful in confirming the presence of bilateral pleural effusions.

\section{REFERENCES}

1. Merritt CRB, Matthews CC, Scheinhorn D, et al: Digital imaging of the chest. J Thorac Imaging 1:1-13, 1985

2. Kangarloo H, Boechat MI, Barbaric Z, et al: Two-year clinical experience with a computed radiography system. AJR 151:605-608, 1988

3. Sonoda M, Takano M, Miyahara J, et al: Computed radiography utilizing scanning laser stimulated luminescence. Radiology 148:833-838, 1983

4. Sherrier RH, Chotas HG, Johnson GA, et al: Image optimization in a computed radiography/photostimulable phosphor system. J Digit Imag 2:212-219, 1989 